\title{
Economic consequences of geotechnical instabilities in open cut coal mines
}

\author{
K Young BHP, Australia
}

A Robotham BHP, Australia

G Virk BHP, Australia

\begin{abstract}
Having a robust reporting culture and a best practice reporting and recording tool has enabled BHP Coal to record and manage over 9,000 geotechnical hazards in their open cut mines over the past 12 years. The good reporting culture and well-defined trigger action response plan (TARP) process indicates that the majority are TARP level 1 hazards, which only require additional monitoring or standoffs. However, some of these hazards have had a significant financial impact on the business. Quantifying the direct and indirect economic consequences of geotechnical hazards on the business is critical to inform appropriate exploration and mine planning decisions. The costing process is complex, particularly regarding the impacts on productivity such as re-scheduling equipment, and temporarily moving to less economic pits.

The geotechnical hazards recorded at BHP's coal mines over two years were costed using a BHP Coal developed tool. Costing included consequences incurred by the business due to geotechnical issues, such as the cost of lost, delayed and diluted coal, equipment remediation and re-scheduling, and delays to the mining schedule.

Investigations into the relationship between the root cause and hazard cost identified geological structure, unachievable design, material strength, and blasting as major contributing factors.

Results indicate that productivity for the business is most sensitive to hazards that result in delayed or abandoned coal. Unrecognised geological structures in the highwall were assessed to have the largest economic consequence at BHP coal mines over the two years, driven by lost coal, delayed coal, diluted coal, and buttressing of highwalls to provide additional stability.
\end{abstract}

Keywords: geotechnical instabilities, economic consequences, coal mines, cost

\section{$1 \quad$ Introduction}

Geotechnical instabilities can have both safety and economic impacts. There are often significant economic costs associated with geotechnical instabilities and hazards. However, these costs are not routinely calculated and recorded across the mining industry but are in the magnitude of millions to tens of millions of dollars per site per year.

Mining companies aim for superior returns for their shareholders and accordingly are seeking innovative methods of improving economic efficiency.

One opportunity for mining companies to improve business performance is to optimise the balance between investment in geological exploration within the five year mining plan and the acceptance of geotechnical risks. Maximised exploration and resultant increase in confidence of the geological model allows geotechnical engineers to produce designs that minimise or reduce geotechnical risk from the mine plan.

Reduced exploration and thus lower confidence in the geological model can result in either more conservative and costly geotechnical design, or in the case of unknown structure, not mitigating the structure in design. This leads to more geotechnical hazards and higher cost of remediation to ensure the environment is suitably safe while maximising extraction of the coal resource. 


\section{Reporting hazards at BHP}

BHP Coal implemented an electronic reporting system to record and track geotechnical, operational and environmental hazards in 2007. Open cut examiners have reported over 75,000 hazards from 2007-2019, 9,900 of which were or are geotechnical hazards (as of October 2019). GPS coordinate centres for hazards have been recorded since the inception of the electronic reporting tool in 2007. The spatial location and extent of all hazards has now been integrated following a system upgrade in March 2019. This electronic system is referred to as the Hazard Reporting Tool (HazRT).

Information recorded in the hazard reporting systems has always included generic location (e.g. highwall, low wall or truck dump), and operational conditions such as the controls put in place and access to the hazardous area. In addition, the geotechnical hazards are classified by trigger action response plan (TARP) level. A TARP Level 1 hazard is considered a minor geotechnical hazard, TARP Level 2 is where a major geotechnical hazard or situation requires geotechnical inspection and issuing a hazard report, and TARP Level Failure represent hazards that breached controls, affecting an area outside the recommended stand-off or safe working distance. TARP Level Failures likely lead to a formal investigation to prevent reoccurrence.

Once a major TARP Level 2 or TARP Level Failure geotechnical hazard is entered into the system by the open cut examiner, BHP Coal geotechnical engineers use a dedicated geotechnical reporting tool to enter additional geotechnical information such as the failure mechanism, root cause, additional controls, and geotechnical hazard report. The information entered by the open cut examiners and the geotechnical engineers delivers an auditable record of geotechnical hazards that can be analysed for trends such as reoccurring mechanisms, repeating spatial locations, and root causes.

\section{BHP Coal Cost Calculator}

Accurate estimates of the costs associated with geotechnical hazards in the open cut coal mining industry have not been formally reported to date. In 2018, the BHP Coal Cost Calculator was developed to establish an understanding of the magnitude of the cost associated with geotechnical hazards in coal mines and key contributors to the cost of the hazards. The long-term aim of the calculator was to compare the average cost of hazards to both the cost of mitigation in advance of design changes, as well as the requests for additional exploration to improve design assumptions.

To calculate the cost of hazards, using the calculator, the primary factors were the costs associated with the remediation of hazards to make the environment safe allowing mining to continue, sterilisation of mineable coal, long-term delay to the mining of coal, and equipment standby. The calculator was built with the functionality to cost geotechnical design changes, for example additional blasting, to mitigate geotechnical hazards.

The main drivers in remediating geotechnical hazards are to make the mine environment safe for coal mine workers and enable an efficient return to mining where possible. There are many different methods used to remediate areas, including, but not limited to, establishing a physical bund around the area, wall scaling, removal of failed material, or implementation of a buttress.

Given the scale of some geotechnical hazards, not all coal can be mined safely or productively. In these cases, coal is sterilised from the mine plan. The calculator accounts for the value of this coal including loss through dilution. In some cases, coal can be safely recovered after remediation or at a later time in the mining process. Delayed coal mining causes a deferral of profits and a lower return rate for the investment of overburden removal. To account for the cost of delayed coal, the calculator uses a net present value formula.

Equipment delays formed another identified cost associated with geotechnical hazards. The cost of equipment being parked was viewed in two ways with the following assumptions for each case: for non-critical work, the cost was viewed to be the sunk cost associated with the equipment that was being spent without returning any value; for critical work, it was assumed that the deficit would be absorbed by contract fleets, with the calculator using contract unit rates. 
Equipment production rates, value and yield of coal and equipment running costs vary by site. In order to improve accuracy, inputs were entered for individual sites included in the calculator with the option to nominate the particular site when completing the costing of hazards.

Due to the complex nature of costing hazards there are some limitations to the cost calculator tool. One of the most significant challenges associated with the tool was how to calculate lost opportunity costs and hazards that may have a flow-on effect. Examples include delayed production of coal that is critical to coal blending as opposed to coal that is stockpiled for later use and the schedule impacts due to equipment productivity where equipment is shut down as opposed to kept running but moved onto lower priority planned work. Cases like this had to be examined using simplifications within the bounds of the calculator using defined unit rates with conventional scenarios and may not illustrate the full extents of the cost, particularly when schedules were impacted.

\section{$4 \quad$ Hazard cost calculation}

The individual cost of geotechnical hazards from BHP's nine active coal mines over a past two year period was estimated using the BHP Coal Cost Calculator, resulting in data for 384 hazards with a varying magnitude of impact. This involved extracting details from each hazard including pit location, root cause, failure mechanism, remediation executed and impact on the mine plan.

\subsection{Hazard root cause classification}

The root cause of each geotechnical instability was classified as being primarily caused by one of the categories listed in Table 1, using information from HazRT, the geotechnical engineer's hazard reports, and direct observation of root cause where available. In some cases, a secondary or tertiary root cause was also defined.

Table 1 Root cause definition for geotechnical instabilities

\begin{tabular}{|c|c|}
\hline Root cause & Root cause definition \\
\hline $\begin{array}{l}\text { Geological } \\
\text { structures }\end{array}$ & $\begin{array}{l}\text { Instability caused by geological structure that was unknown or different from the } \\
\text { geological or structural model. The mine design has not been adapted to mitigate the } \\
\text { effects of the structure leading to instability. }\end{array}$ \\
\hline $\begin{array}{l}\text { Material } \\
\text { strength }\end{array}$ & $\begin{array}{l}\text { Where variations occurring in the actual rock strength in comparison to } \\
\text { expected/modelled parameters lead to an instability. }\end{array}$ \\
\hline Water & $\begin{array}{l}\text { Instabilities induced by the presence of surface or groundwater. These could be related } \\
\text { to poor understanding of the watertable and impacts of weather. }\end{array}$ \\
\hline $\begin{array}{l}\text { Unachievable } \\
\text { design }\end{array}$ & $\begin{array}{l}\text { Instabilities caused when the geotechnical/mine design cannot be achieved } \\
\text { operationally. This likely occurs when the design specification did not consider mining } \\
\text { or equipment compatibility. }\end{array}$ \\
\hline Blasting & $\begin{array}{l}\text { Damage to the rock mass caused by drilling and blasting activities, which leads to } \\
\text { instabilities. }\end{array}$ \\
\hline $\begin{array}{l}\text { Operational - } \\
\text { water/mud }\end{array}$ & $\begin{array}{l}\text { Instabilities caused by the inadequate management of surface water and presence of } \\
\text { mud. This could be related to allowing water to pool, inadequate drainage and water } \\
\text { management, as well as mud initiating weak foundation conditions. }\end{array}$ \\
\hline $\begin{array}{l}\text { Non- } \\
\text { compliance to } \\
\text { design }\end{array}$ & $\begin{array}{l}\text { Mine design is often informed by geotechnical models. Instabilities can be caused by pit } \\
\text { operations deviating from the mine design causing it to be outside what was } \\
\text { geotechnically assessed as stable. }\end{array}$ \\
\hline
\end{tabular}




\section{$5 \quad$ Results}

The resultant financial data collected from the geotechnical hazards allowed for an evaluation determining leading cost drivers, and hazard area with the costliest and most prominent root cause contributing to total cost.

\subsection{Cost drivers}

The BHP Coal Cost Calculator breaks instability cost down into a number of different cost items. The dominant cost drivers associated with geotechnical instabilities were classified into six categories as listed in Table 2 .

Table 2 Cost driver definitions for geotechnical instabilities

\begin{tabular}{|c|c|}
\hline Cost driver & Cost driver definition \\
\hline Sterilised coal & $\begin{array}{l}\text { Sterilised coal is when coal initially planned to be mined is abandoned when a } \\
\text { geotechnical hazard prevents mining the coal safely or economically. }\end{array}$ \\
\hline Delayed coal & $\begin{array}{l}\text { Coal is delayed when the presence of a geotechnical hazard makes it unsafe to } \\
\text { continue mining. The remaining coal is then safely recovered in following strips. } \\
\text { The financial benefits of mining the coal is also delayed, which has economic } \\
\text { consequences for the business. }\end{array}$ \\
\hline Diluted coal & $\begin{array}{l}\text { Coal is diluted when failed material mixes with the coal resource, resulting in coal } \\
\text { being sterilised. }\end{array}$ \\
\hline $\begin{array}{l}\text { Removing failed } \\
\text { material }\end{array}$ & $\begin{array}{l}\text { Where a hazard results in failed material in the pit, such material must be removed } \\
\text { before mining operations can safely continue. Generally, this material is removed } \\
\text { with truck and shovel equipment. It results in a loss of productivity as the } \\
\text { equipment has to deviate from the optimised mine plan to remove failed material. }\end{array}$ \\
\hline $\begin{array}{l}\text { Buttressing } \\
\text { highwall }\end{array}$ & $\begin{array}{l}\text { A fill buttress can be implemented to add additional resisting forces to a hazard } \\
\text { that is, or is likely to, move. Buttress size and material is recommended by a } \\
\text { geotechnical engineer and installed by truck and shovel. Costs are mainly } \\
\text { associated with loss of productivity of equipment installing the buttress. }\end{array}$ \\
\hline $\begin{array}{l}\text { Dragline } \\
\text { standby }\end{array}$ & $\begin{array}{l}\text { Where it is unsafe for the dragline to continue operations, a dragline standby cost } \\
\text { is incurred as a sunk cost that does not return value. In contrast to other mining } \\
\text { equipment that can be directed to alternative mining locations, a dragline has } \\
\text { restricted mobility that inhibits short-term deviation from the mine plan. }\end{array}$ \\
\hline
\end{tabular}

Figure 1 shows the cost drivers as a percentage of the cost to the business over the past two years. Abandoned coal was the most significant driver of geotechnical hazard costs over the two years, contributing $60 \%$ of the total geotechnical hazard costs. These results indicate that productivity for the business is most sensitive to hazards that delay or abandon coal mining, as they combine to $72 \%$ of the total cost of geotechnical hazards. 


\section{Significant Drivers of Hazard Cost}

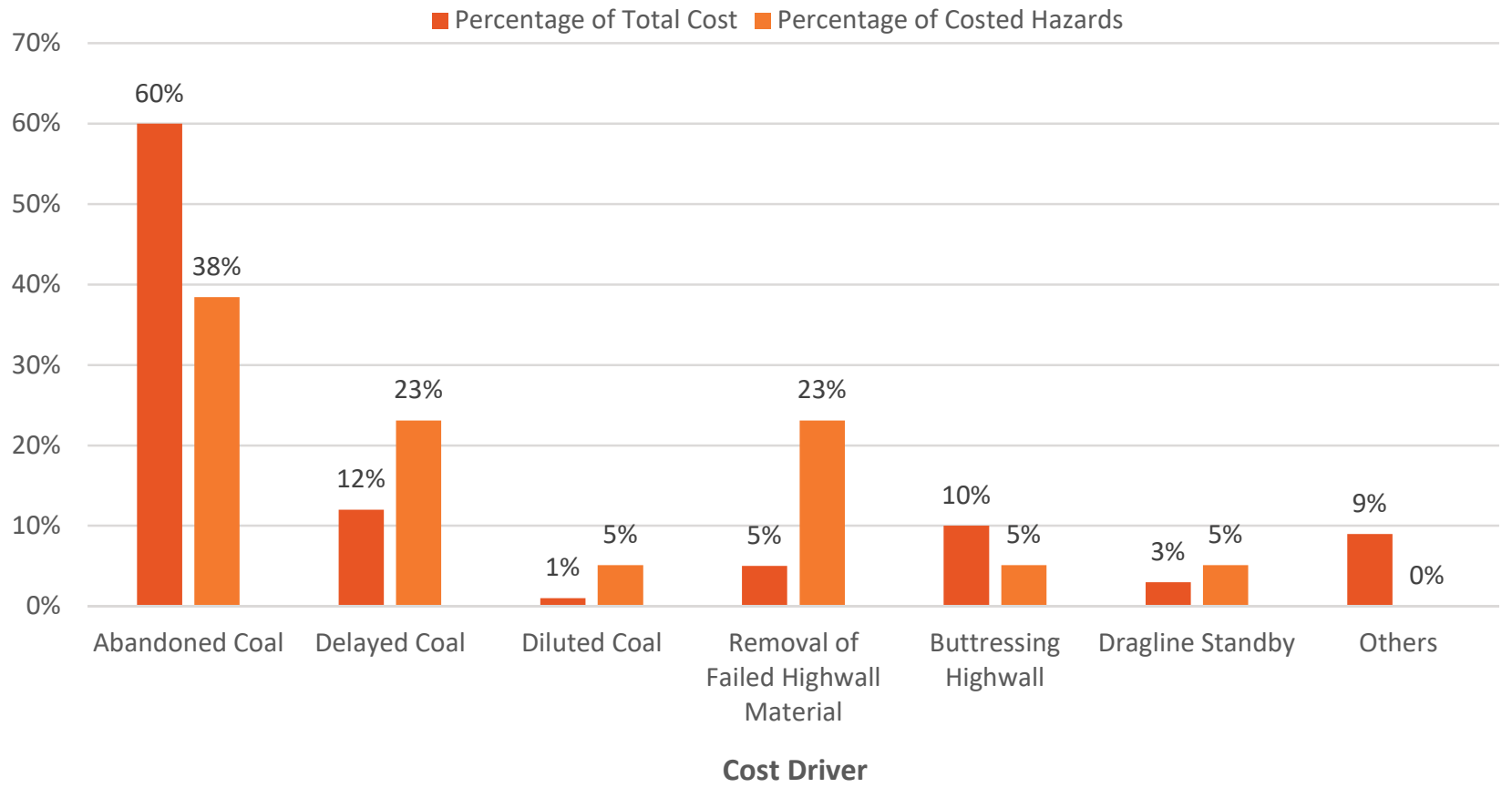

Figure 1 Cost drivers of geotechnical hazard costs by percentage and count of costed hazards

\subsection{Hazard location}

The contribution to the total economic consequences of geotechnical hazards varied significantly given the hazard's location within the mine. The hazard location is classified in HazRT as a highwall/endwall instability, low wall instability, dragline bench instability or a dump instability. As seen in Figure 2, highwall and endwall hazards contributed $95 \%$ of the total geotechnical hazard costs over the two year analysis period.

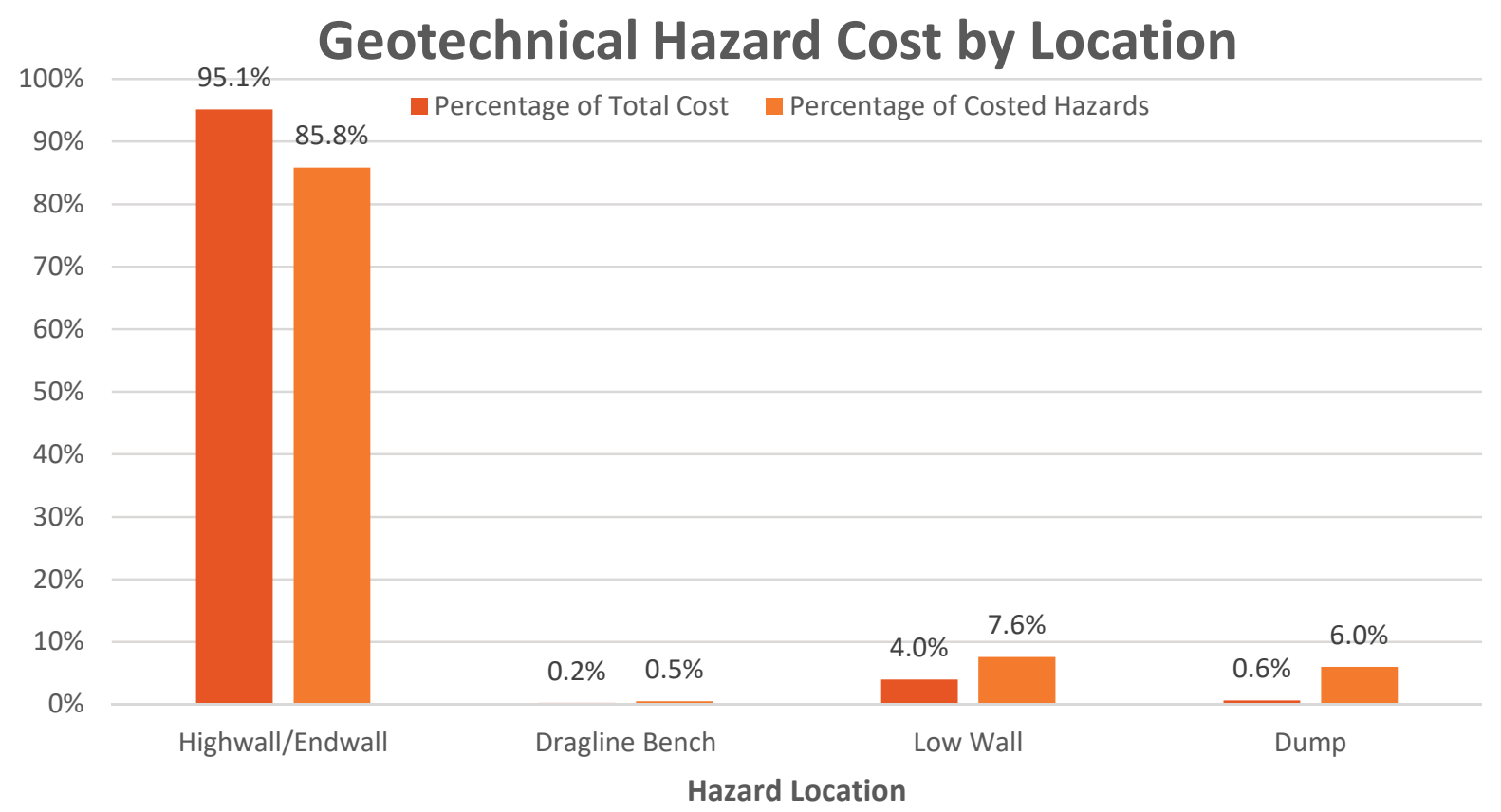

Figure 2 Location of geotechnical hazard costs by percentage and count of costed hazards 
Highwall instabilities often lead to coal being abandoned or delayed, which is the reason for the significance of the cost of these instabilities. Remediating low wall, dragline bench and dump instabilities was not as strongly correlated to coal loss.

\subsection{Root cause of highwall hazards}

The root cause of each individual hazard is determined at the time of hazard reporting. Figure 3 shows the proportion of highwall instabilities associated with each root cause. Statistics relating to root causes from the current dataset are discussed in this section.

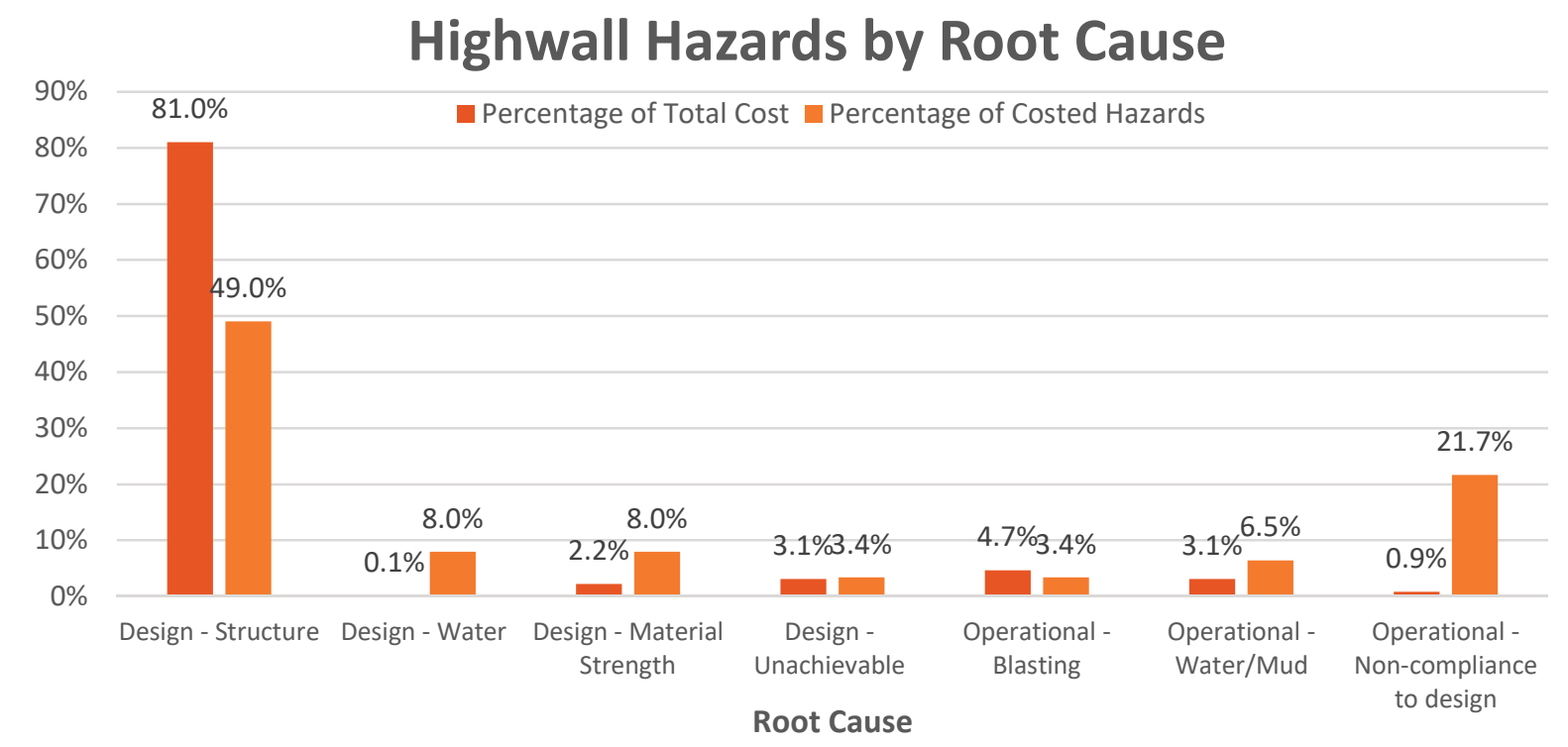

Figure 3 Proportion of highwall hazards with respect to their root cause

\subsubsection{Design - structure}

Instabilities caused by geological structures that were unknown and therefore not considered in the highwall design contributed approximately $81 \%$ of the total hazard cost in highwalls over two years. An additional $2 \%$ of total geotechnical instability costs arose from unidentified geological structures acting as a secondary or tertiary root cause. In these cases, the presence of geological structures exacerbated the impact of the primary root cause.

\subsubsection{Design - material strength}

While, as a primary root cause, material strength only made up $2.2 \%$ of highwall hazards, $55 \%$ of total geotechnical instabilities identified material strength as a secondary root cause. The presence of weaker material heightened the impact of the primary root causes, particularly unidentified geological structures. The results of this investigation illustrate that buttressing to provide additional stability is the most common remediation required as a consequence of incorrectly defined material strength.

\subsubsection{Water - design and operational}

Water does not significantly contribute to the overall cost of geotechnical hazards as a primary root cause. The primary cost driver for water-related hazards is equipment being placed on standby, generally a small cost when compared to other remediation methods. However, an additional $8 \%$ of total geotechnical instability costs arise from watertable conditions acting as a secondary root cause. In these hazards, the presence of the watertable compounded the impact of other primary root causes, predominantly unidentified geological structures and material strength. 


\subsubsection{Operational - blasting}

Geotechnical hazards induced by blasting account for the second highest percentage of highwall instabilities. The results of this investigation illustrate that highwall instabilities resulting from blasting operations have the potential to cause coal to be delayed to following strips or coal to be abandoned. The amount of coal delayed or abandoned is on average less than what is seen from a structural-related failure, which results in a lower contribution to the overall total cost of instabilities.

\subsubsection{Operational - non-compliance to design}

Non-compliance to design is one of the lower contributors to cost by root cause, however, it is the second highest cause by count after structural instability making up $17 \%$ of all highwall hazards. The largest contributors to cost from non-compliance are buttressing for additional stability and highwall scaling to remove rockfall risk. Bund installation is used to control instabilities in $86 \%$ of hazards caused by non-compliance to design. Bund installation in particular is of minor cost when compared to other remediation methods.

\section{Discussion}

The data suggests that there are significant financial savings to be made by considering the dominant cost drivers for geotechnical hazards. The review has identified key areas that could be de-risked to lower costs associated with hazards and increase coal recovery.

Abandoned coal and delayed coal was determined to be the largest area of cost associated with geotechnical instabilities and hazards. Coal was abandoned as a result of geotechnical hazards when:

- The hazards prevented safe access to the pit.

- The hazards did not allow safe operations to occur within the pit.

- The hazards buried coal under failed rock material, and separation of the rock material from the coal was either unsafe or uneconomical.

If the number and severity of these types of hazards are prevented or mitigated, there would be a significant reduction in the cost associated with geotechnical hazards. Highwall structural-related hazards are the hazard type that most strongly correlated to a subsequent delay or abandonment of coal.

Unexpected cost and associated delays with remediating hazards can also have flow-on effects which cannot be determined using the calculator as they are often case-specific. Achieving plan stability by decreasing geotechnical hazards allows for more effective planning and maximising equipment utility and coal recovery. This can be achieved in mainly two ways: structural model considerations in the slope design and compliance to design.

Whilst the location has been tracked in HazRT, there is no historical database tracking the size of the hazard. The functionality to identify the extents of the hazard has been built in to recent upgrades of the system, so this may be available moving forward. There is almost certainly a link between the size of the hazard and the cost of the remediation, and likewise the extent of the hazard with the root cause of the hazard, however, this information was not recorded.

\subsection{Structural instability}

Structural failures usually refers to instabilities associated with geological structure; in particular, faults and joints leading to wedge, planar and toppling failures. To control geotechnical instabilities associated with structure, faults and joints must be considered in pit design so that pit walls can be adapted in orientation, angle and design (Department of Minerals and Energy Western Australia 1999). Unidentified and incorrectly identified geological structures can pose economic threats to a mining business (Hanson et al. 2005) as the unidentified structures are not considered in the mine plan and, therefore, the mine plan is not designed to 
mitigate their effects. The results of this investigation illustrate that highwall instability as a result of unidentified geological structures have significant economic consequences to a mining business.

Although structural-related hazards represent less than $50 \%$ of highwall hazards, they make up over $80 \%$ of the cost. This indicates that structural-related hazards are more likely to be of a higher consequence and magnitude and lead to impacts that are more costly, including coal loss and coal delay.

Geological models are sometimes developed with primary focus of resource estimation and quality. Widening scope to ensure that geotechnical modelling includes an adequate structural model with the intent of geotechnical engineers as the end user and the purpose of describing "the orientation and spatial distribution of the structural defects that are likely to influence the stability of pit slopes" (Read \& Stacey 2009 , p. 69). Achieving high levels of geological confidence to account for structure persistence and orientation allows geotechnical engineers to mitigate the impact of structure on slope designs and associated mine plans. This can be achieved by investment in geological exploration, modelling, and mapping, as well as increased engagement between geological modellers and geotechnical engineers.

Structural instability was found to compound the impact and magnitude of hazards with other primary root causes in particular blasting, material strength and water. Improvements or a focus on a structural model as opposed to a geological resource model will also have flow-on beneficial effects for blasting, allowing blasting to be optimised around structure. Understanding the interaction between material strengths and structure as well as material strengths and porewater pressure would also provide higher confidence in geotechnical design.

\subsection{Compliance to design}

Compliance to design was found to be a major contributor to the number of geotechnical hazards. Compliance to design was determined as the root cause of $17 \%$ of geotechnical hazards and $22 \%$ of highwall hazards. However, the cost of these hazards was less than $1 \%$ of the total cost of highwall hazards. This was linked to the lower magnitude of related hazards and resultant remediation costs generally being less significant as they did not cause coal loss or delay.

Although it is important that compliance to design is focussed on from a safety perspective and appropriate controls should be put in place when hazards do occur, it is not a large contributor to the overall financial cost of geotechnical hazards.

\section{Conclusion}

A strong geotechnical reporting culture and a custom cost calculator allowed the financial cost of 384 geotechnical hazards on BHP's coal business to be calculated. Hazards were classified according to their root cause, location, and cost drivers.

Productivity for the business is most sensitive to hazards that delay or abandon coal mining, contributing $72 \%$ of total hazard cost over two years. $95 \%$ of geotechnical hazard costs incurred by the business are located at highwalls and endwalls.

Hazards associated with unidentified geological structures represent less than $50 \%$ of highwall hazards, however are associated with over $80 \%$ of the financial costs of these hazards. Structure-related hazards were more costly than other hazards because they are of a higher consequence and magnitude than hazards caused by other root causes, prevent safe operational access to the pit, and cause coal abandonment or delay.

The results from this investigation indicate that the most effective financial gains can be realised by increasing levels of confidence in geological structural models through investment in geological exploration, modelling and mapping to reduce the magnitude and consequence of highwall hazards.

BHP has begun to incorporate the costs that have been attributed to structural instabilities into geological and geotechnical risk assessments. This allows the assignment of actual values for potential losses based on historical events and aids the cost-benefit analysis to justify the requirement for additional drilling. 
As exploration drilling is most often in the 3-5 year planning space, seeing the benefits of additional geological and improved structural information informing design will likely flow through in the years to come.

The cost calculator is currently being used at BHP in the geotechnical risk assessment to estimate hazard cost during the mine planning phase. This has helped to inform assessments regarding adapted designs to remove the hazard before it occurs and help with decisions associated with remediation options.

\section{Acknowledgement}

The BHP Coal Cost Calculator and upgrade to electronic hazard reporting were initiatives driven by BHP Coal's geotechnical services manager, Dan Payne. Dan's motivation was to understand the cost of geotechnical hazards for the purpose of justifying the cost of exploration and to enable the appropriate business decision between design changes and hazard mitigation. The authors thank Dan for his encouragement and inspiration through the development, investigation, and writing processes.

The authors are grateful to BHP Coal for permission to publish the paper and present at this conference. They also acknowledge the significant effort by other employees of BHP Coal to create the BHP Coal Cost Calculator, particularly Warren Hitchcock as the co-creator of the Cost Calculator and valued editor, other members of Geotechnical Services, and mine planning engineers for site-specific inputs.

\section{References}

Department of Minerals and Energy Western Australia 1999, Geotechnical Considerations in Open Pit Mines, viewed 1 February 2019, http://www.dmp.wa.gov.au/Documents/Safety/MSH_G_GeotechnicalConsiderationsOpenPitMinespdf.pdf

Hanson, C, Thomas, D \& Gallagher, B 2005, 'The value of early geotechnical assessment in mine planning', in N Aziz (ed.), Proceedings of Coal 2005: Coal Operators Conference, University of Wollongong, Wollongong, and The Australian Institute of Mining and Metallurgy, Carlton, pp. 17-30.

Read, J \& Stacey, P 2009, Guidelines for Open Pit Slope Design, CSIRO Publishing, Collingwood. 
